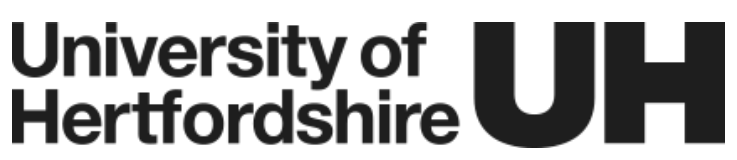

\section{Citation for the published version:}

Luo, Q., Sun, Y., Li, P., Simpson, O., Tian, L., \& He, Y. (2018). Generalized

Completed Local Binary Patterns for Time-Efficient Steel Surface Defect

Classification. IEEE Transactions on Instrumentation and Measurement. DOI:

10.1109/TIM.2018.2852918

Document Version: Accepted Version

\section{Link to the final published version available at the publisher:}

\section{https://doi.org/10.1109/TIM.2018.2852918}

(C) 2018 IEEE. Personal use of this material is permitted. Permission from IEEE must be obtained for all other uses, in any current or future media, including reprinting/republishing this material for advertising or promotional purposes, creating new collective works, for resale or redistribution to servers or lists, or reuse of any copyrighted ncomponent of this work in other works.

\section{General rights}

Copyright $\odot$ and Moral Rights for the publications made accessible on this site are retained by the individual authors and/or other copyright owners.

Please check the manuscript for details of any other licences that may have been applied and it is a condition of accessing publications that users recognise and abide by the legal requirements associated with these rights. You may not engage in further distribution of the material for any profitmaking activities or any commercial gain. You may freely distribute both the url (http://uhra.herts.ac.uk/) and the content of this paper for research or private study, educational, or not-for-profit purposes without prior permission or charge.

\section{Take down policy}

If you believe that this document breaches copyright please contact us providing details, any such items will be temporarily removed from the repository pending investigation.

\section{Enquiries}

Please contact University of Hertfordshire Research \& Scholarly Communications for any enquiries at rsc@herts.ac.uk 


\title{
Generalized Completed Local Binary Patternsfor Time-efficient Steel Surface Defect Classification
}

\author{
Qiwu Luo, Member, IEEE, Yichuang Sun, Senior Member, IEEE, Pengcheng Li, Oluyomi Simpson, \\ Member, IEEE, Lu Tian, and Yigang He, Member, IEEE
}

\begin{abstract}
Efficient defect classification is one of the most important preconditions to achieve online quality inspection for hot-rolled strip steels. It is extremely challenging owing to various defect appearances, large intra-class variation, ambiguous inter-class distance, and unstable gray values. In this paper, a generalized completed local $b$ inary patterns (GCLBP) framework is proposed. Two variants of improved completed local binary patterns (ICLBP) and improved completed noise-invariant local-structure patterns (ICNLP) under the GCLBP framework are developed for steel surface defect classification. Different from conventional LBP variants, descriptive information hidden in nonuniform patterns is innovatively excavated for better defect representation. This work focuses on the following aspects: First, a lightweight searching algorithm is established for exploiting the dominant nonuniform patterns (DNUPs). Second, a hybrid pattern code mapping mechanism is proposed to encode all the uniform patterns and DNUPs. Third, feature extraction is carried out under the GCLBP framework. Finally, histogram matching is efficiently accomplished by simple nearest neighbor classifier (NNC). The classification accuracy and time-efficiency are verified on a widely recognized texture database (Outex) and a real-world steel surface defect database (NEU). The experimental results promise that the proposed method can be widely applied in online AOI instruments for hot-rolled strip steel.
\end{abstract}

Index Terms-Surface defects, image classification, hot-rolled strips, local binary patterns (LBP), automatic optical inspection (AOI) instrument.

\section{INTRODUCTION}

$\mathrm{H}$ OT-ROLLED strip steel occupies more than $50 \%$ of all the products in iron and steel industry. It is not only the essential raw material of cold-rolled steel, but also acts as vital material in architecture, machinery and automobile industries. Any untimely solved defect would lead to huge economic and reputation losses to steel manufacturers. Online surface defect

Qiwu Luo is with the School of Electrical Engineering and Automation, Hefei University of Technology, Hefei, China. (Corresponding Author, Qiwu Luo, Email: luoqiwu@hfut.edu.cn).

Yichuang Sun and Oluyomi Simpson are with the School of Engineering and Technology, University of Hertfordshire, Hatfield, Herts AL10 9AB, United Kingdom.

Pengcheng Li is with the School of Automobile and Traffic Engineering, Hefei University of Technology, Hefei, China.

$\mathrm{Lu}$ Tian is with the Powder Metallurgy Research Institute, Central South University, Changsha, China, he is also with RAMON Beijing Research Institute, RAMON Science \& Technology Co., Ltd., Beijing, China.

Yigang He is with the School of Electrical Engineering, Wuhan University, Wuhan, China, he is also with the School of Electrical Engineering and Automation, Hefei University of Technology, Hefei, China. inspection of hot-rolled strip steel is thus extremely significant. It is mainly handled by an automatic optical inspection (AOI) instrument. In general, defect detection and defect classification are two main functions of AOI instruments [1]-[8]. The former is merely to detect defective regions from massive surface images. It is the foundation of the "quality problem close loop", as early defect inspection will result in fewer losses. The latter is to identify the defect categories. It is used for grading and distributing end products. The detailed flow chart is illustrated in Fig. 1. Consequently, defect detection and classification with high time-effectiveness are the essential preconditions of online quality control.

Defect detection based on supervised or unsupervised learning methods becomes mature as the rapid development of computer vision and pattern recognition. Ghorai et al. proposed a typical supervised learning algorithm- VVRKFA [6]. It was then successfully applied to the AOI instrument for hot-rolled flat steel. A true positive rate (TPR) of $93.8 \%$ with an upper limit of $5 \mathrm{~m} / \mathrm{s}$ rolling speed was achieved [3]. Luo et al. developed a cost-effective hot-rolled steel AOI instrument. It was driven by hardware acceleration technique and the upper limit of rolling speed was elevated to $20 \mathrm{~m} / \mathrm{s}$ [4]. Liu et al. constructed an unsupervised Haar-Weibull-variance (HWV) model, and a higher TPR of $96.2 \%$ was achieved [7].

Although defect detection and defect classification are inseparable in AOI instrument, the room for defect classification improvement is large. The following are challenges which defect classification has to handle:

1) Large intra-class variation and ambiguous inter-class distance. For example, the outlines of patches shown in Fig. 11(b) are diverse, while the crazing in Fig. 11(c) and pitted surface in Fig. 11(d) have similar appearance.

2) Unstable gray values. The gray value is sensitive to cooling water [4], uneven illumination [5], multiple material types [8] and mechanical vibration [3], [9]. As shown in Fig. 11, the gray values have giant difference in the 6 defect classes.

3) Limited image classification time. Time-efficient defect classification is of benefit to online quality control and effective production increase.

Accordingly, it is difficult to classify these defects simply by gray statistics or threshold judgments. Various algorithms such as MGA [10], Bayes theorem [11], SVM [12], the aforementioned VVRKFA [6] and the latest CNN [13] can be used for feature extraction and matching. However, compared with time-efficiency, most of algorithms pay more attention to classification accuracy, while time-efficiency is a key performance indicator which determines whether new methods could be applied in industrial manufacture. 


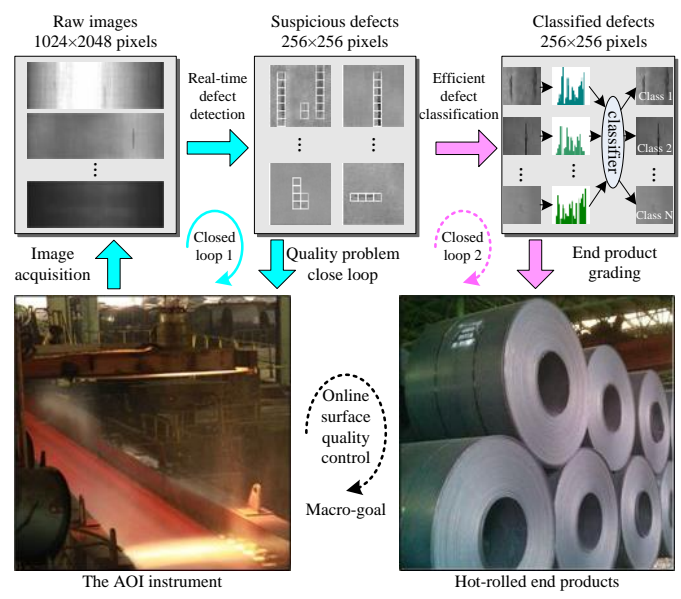

Fig. 1. Brief technical routes of AOI instrument. Two closed loops (marked green and red) jointly support achieving online surface quality control.

This paper investigated that the local binary patterns (LBP) descriptor is lightweight and efficient for texture representation [14]-[16]. This descriptor and its various variants, such as completed LBP (CLBP) [24], completed noise-invariant local-structure pattern (CNLP) [25] and dominant LBP (DLBP) [26], have been successfully applied to face recognition [17]-[19], texture classification [20], [21] and visual tracking [22]. Some literature on surface defect inspection based on LBP are reported [8], [23].

This paper focuses on defect classification (closed loop 2 in Fig. 1). A generalized CLBP (GCLBP) framework is proposed for further developing image-based AOI measurements. LBP pattern information and frequency information are considered simultaneously, and the noise robustness is promoted implicitly during the dominant nonuniform pattern pursuing process. The contributions are as follows:

1) A concealed yet objective fact that nonuniform patterns contain useful descriptive information is discovered. The number of nonuniform patterns is less than that of uniform patterns, but nonuniform patterns are not negligible in improving classification accuracy.

2) A novel GCLBP framework is proposed to innovatively excavate the implicit descriptive information from nonuniform patterns. The overall performance of CLBP-like descriptors (CLBP and CNLP for case study) is further strengthened.

3) The classification accuracy and time-efficiency of two typical GCLPB-based variants, i.e., ICLBP and ICNLP, have been successfully verified on both a widely used texture database (Outex [28]) and an actual steel surface defect database (NEU [29]).

The rest of this paper is organized as follows. Section II includes the related theory preliminaries and the initial research motivation. Section III elaborates the proposed GCLBP framework in detail. Section IV highlights the novelty and advantages of GCLBP. Extensive experiments and related discussions are demonstrated in Section V. Finally, Section VI concludes this research.

\section{PRELIMINARIES AND MOTIVATION}

The proposed GCLBP framework is basically generalized from CLBP. Here, the LBP, CLBP, and CNLP (a CLBP-based variant) are reviewed briefly.

\section{A. Review of $L B P$}

Given a central pixel $g_{c}$ and its $P$ circularly spaced neighbors $g_{p}$ with radius $R$, there exits positional index array $p, p=0, \ldots$, $P-1$. The original LBP code of $g_{c}$ can be simply calculated by

$$
L B P_{P, R}=\sum_{p=0}^{P-1} 2^{P} s\left(d_{p}\right), d_{p}=g_{p}-g_{c}
$$

where $s(t)=(\mathrm{t} \geq 0) ? 1: 0$, it is a simple sign function. The gray values corresponding to out-of-center coordinates are solved by bilinear interpolation [27]. An evaluation criterion of pattern uniformity $U()$ which reflects the spatial transitions between bitwise ' 0 ' and ' 1 ' has been defined as

$$
\left\{\begin{array}{l}
U\left(L B P_{P, R}\right)=U_{h 2 t}\left(L B P_{P, R}\right)+U_{\text {intrm }}\left(L B P_{P, R}\right) \\
U_{h 2 t}\left(L B P_{P, R}\right)=\left|s\left(g_{P-1}-g_{c}\right)-s\left(g_{0}-g_{c}\right)\right| \\
U_{\text {intrm }}\left(L B P_{P, R}\right)=\sum_{p=1}^{P-1}\left|s\left(g_{p}-g_{c}\right)-s\left(g_{p-1}-g_{c}\right)\right|
\end{array}\right.
$$

where $U_{h 2 t}\left(\right.$ ) and $U_{\text {intrm }}($ ) respectively stand for the head-to-tail and intermediate spatial transitions of the raw LBP codes. Then, uniform patterns and rotation invariant uniform patterns are defined as

$$
\begin{gathered}
L B P_{P, R}^{u 2}=\left\{\begin{array}{cc}
\sum_{p=0}^{P-1} s\left(g_{p}-g_{c}\right) & U\left(L B P_{P, R}\right) \leq 2 \\
L B P_{P, R} & \text { otherwise }
\end{array}\right. \\
L B P_{P, R}^{r i u 2}=\left\{\begin{array}{cc}
\sum_{p=0}^{P-1} s\left(g_{p}-g_{c}\right) & U\left(L B P_{P, R}\right) \leq 2 \\
P+1 & \text { otherwise }
\end{array}\right.
\end{gathered}
$$

Hence, the pattern labels based on $L B P_{P, R}^{u 2}$ or $L B P_{P, R}^{\text {riu } 2}$ are decreased to $P \times(P-1)+3$ and $P+2$ from the previous $2^{P}$. The transformations between these different pattern codes can be realized flexibly through simple lookup tables.

\section{B. Review of CLBP}

The $d_{p}$ in (1) is decomposed into $s_{p}$ and $m_{p}$ in CLBP.

$d_{p}=s_{p} \times m_{p}, s_{p}=\operatorname{sign}\left(d_{p}\right)$ and $m_{p}=\left|d_{p}\right|$

where $s_{p}$ is the sign of $d_{p}$ and its expression is $s_{p}=\left(d_{p} \geq 0\right)$ ? $1:-1$, and $m_{p}$ is the magnitude of $d_{p}$. Three CLBP operators -sign, magnitude, and centre pixel operator- are defined as

$$
\begin{aligned}
& C L B P_{-} S_{P, R}=\sum_{p=0}^{P-1} s\left(s_{p}, 0\right) 2^{P} \\
& C L B P_{-} M_{P, R}=\sum_{p=0}^{P-1} s\left(m_{p}, c_{m}\right) 2^{P} \\
& C L B P_{-} C_{P, R}=s\left(g_{c}, c_{I}\right)
\end{aligned}
$$

where $s(t, c)=(t \geq c) ? 1: 0$, it is a combinatorial sign function, $c_{m}$ is the mean value of $m_{p}$ taken over the entire image, and $c_{I}$ is the average gray level of the entire image. In fact, $C L B P_{-} S_{P, R}(6)$ is exactly $L B P_{P, R}(1)$. Two rotation invariant variants are defined

$$
\begin{aligned}
& C L B P_{-} S_{P, R}^{r i}=\min \left\{R O R\left(C L B P_{-} S_{P, R}, i\right) \mid i \in[0, P-1]\right\} \\
& C L B P_{-} M_{P, R}^{r i}=\min \left\{R O R\left(C L B M_{-} S_{P, R}, i\right) \mid i \in[0, P-1]\right\}
\end{aligned}
$$

where $\operatorname{ROR}(x, i)$ is a bitwise cyclic right shift operator. Finally, the pattern dimensions of $C L B P_{-} S_{P, R}^{r i}$ and $C L B P_{-} M_{P, R}^{r i}$ (jointly denoted as $\left.C L B P_{P, R}^{r i}\right)$ are much smaller than $C L B P_{-} S_{P, R}$ and $C L B P \_M_{P, R}$ (jointly denoted as $C L B P_{P, R}$ ).

According to [24], the histograms obtained from CLBP_S, CLBP_M and CLBP_C can be combined to 3-D or 2-D, joint or hybrid histograms, producing sub-variants of CLBP_M/C, CLBP_S/C, CLBP_S/M, CLBP_S_M/C and CLBP_S/M/C. 


\section{Review of CNLP}

To improve noise-robustness, some CLBP variants such as AECLBP [8], CRLBP [36] and CNLP [25] are proposed successively. The key idea of these descriptors is to replace the noise-sensitive threshold of centre pixel value with a more robust compositional variable. The latest CNLP is reviewed here as it is chosen as one of improved cases under the GCLBP framework. The threshold of the sign component is redefined as

$T h_{\text {sign }}=w_{1} g_{c}+w_{2} \frac{g_{c}+\sum_{P=0}^{P-1} g_{p}}{P+1}, w_{1}=w_{2}=0.5$

Then the CNLP_S, CNLP_M and CNLP_C are updated as

$$
\begin{aligned}
& C N L P_{-} S_{P, R}=\sum_{p=0}^{P-1} s\left(g_{p}-T h_{\text {sign }}\right) \\
& C N L P_{-} M_{P, R}=\sum_{p=0}^{P-1} s\left(g_{p}-\left(g_{c}+\frac{1}{P} \sum_{p=0}^{P-1}\left|g_{p}-g_{c}\right|\right)\right) \\
& C N L P_{-} C=s\left(T h_{\text {sign }}-\frac{1}{r \times c} \sum_{i=0}^{r-1} \sum_{j=0}^{c-1} g_{i, j}\right)
\end{aligned}
$$

where $g_{p}, g_{c}$ and $s(t)$ have been defined in (1), and $g_{i, j}$ is the pixel gray value at $i^{\text {th }}$ row and $j^{\text {th }}$ column of the image with size of $r \times c$. Notably, CNLP extract features from centre pixel gray, local intensity and image contrast simultaneously.

\section{Initial Observation and Study Motivation}

Ojala et al. [14], [15] claimed that most of the nonuniform patterns made little contribution to texture representation statistically, since uniform patterns always dominate the prior knowledge of texture images. However, we found a certain quantity of nonuniform patterns have high probability of occurrence in practice. For example, the nonuniform patterns "01000100" and "01000001" appear frequently in $L B P_{8,1}^{r i{ }^{2}}$. In view of this, as shown in Fig. 2, massive statistical tests are conducted on Outex [28] and NEU database [29] to obtain the pattern distribution rule. The proportions of uniform patterns decline significantly with the increment of $P$ and $R$. Furthermore, the $L B P_{8,1}^{r i n 2}$ with the lowest proportion of nonuniform patterns is selected for intuitive presentation in Fig. 3. It can be observed that the boundary of defect (longitudinal entrapped slag) is clearly visible in the right subfigure of Fig. 3(a). Besides, the representation effect of some nonuniform patterns with high probability is even better than that of certain uniform patterns. For instance, the subfigure (Top 20) in Fig. 3(b) shows slightly better representation effect than that of the subfigure (Label=0) in Fig. 3(c).

Traditional LBP variants emphasize only (uniform) pattern information while DLBP only consider frequency information instead of pattern information. These seemingly contradictory viewpoints prompt us to search compelling answers (at least part of them) for the following questions: What kinds of useful descriptive information are implicitly included in nonuniform patterns? How to efficiently exploit the missed information in these nearly ignored nonuniform patterns? And how to maintain high classification accuracy when encountering various textures with loose pattern uniformity degree?

This correspondence attempts to address the above questions by innovatively excavating the dominant nonuniform patterns (DNUPs). A novel defect (also texture) description framework, GCLBP, is thus proposed for efficient image classification.

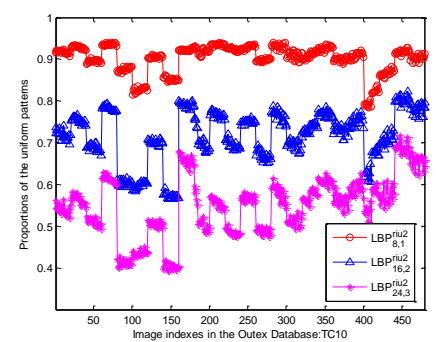

(a) Outex database

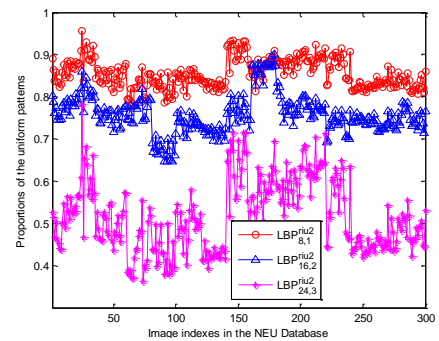

(b) NEU database
Fig. 2. Proportions of the uniform patterns for texture and defective images.

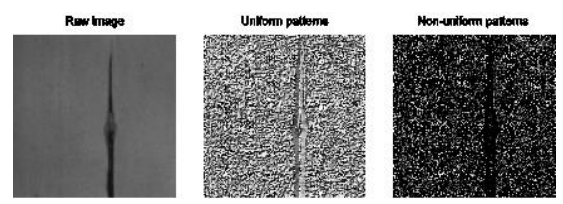

(a) From left to right: raw image, uniform patterns, and nonuniform patterns.

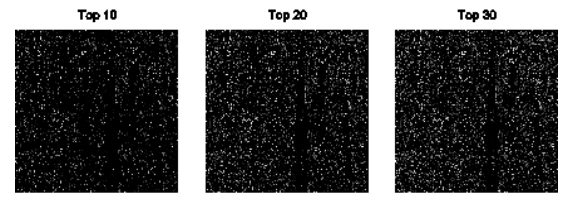

(b) From left to right: Top 10*, Top 20, and Top 30 nonuniform patterns.

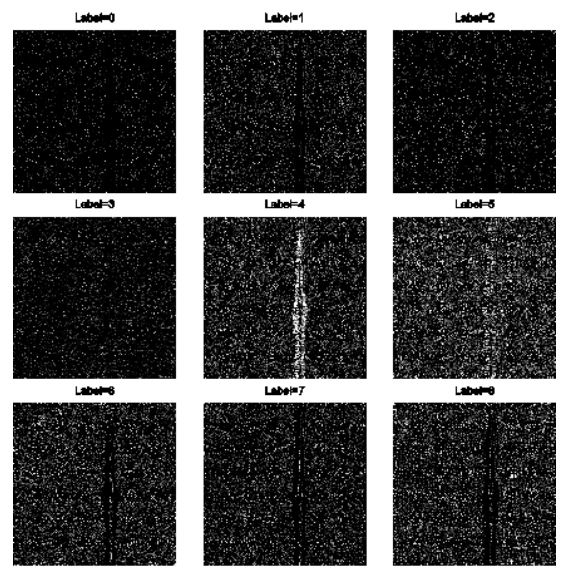

(c) Uniform patterns with 9 distinct pattern labels.

Fig. 3. Representation effects of different $L B P_{8,1}^{\text {riu2 }}$ patterns.

*Note: Top 10 indicates the nonuniform patterns with the highest probability of occurrence and the sum of the probability is $10 \%$. Analogously for Top 20 and Top 30 .

\section{GENERALIZED CLBP (GCLBP)}

\section{A. Dominant Nonuniform Features Pursuing}

The detailed training procedure for excavating dominant nonuniform patterns is presented in Fig. 4. First, given a training set $T\left\{t_{i}[r \times c] \mid i=1,2, \ldots, N_{t}\right\}$ which is constituted of $N_{t}$ images with a size of $r \times c$ pixel, the pattern label of each center pixel in each image is calculated by using a certain CLBP operator (i.e., (6), or (7), or (8)). Second, the calculated pattern labels are discriminatively kept in the buffer pools according to the pattern uniformity defined in (2). Closely after that the pattern label of the last center pixel is obtained, the nonuniform histogram of each image is calculated. The above two steps are carried out for $N$ times to enhance the generalization ability of trained DNUPs. Finally, pattern labels with higher occurrence frequency are selected as DNUPs for the next feature extraction. After repeating test and continuous verification, the range of $\sigma$ 
is recommended to $20 \%-60 \%$.

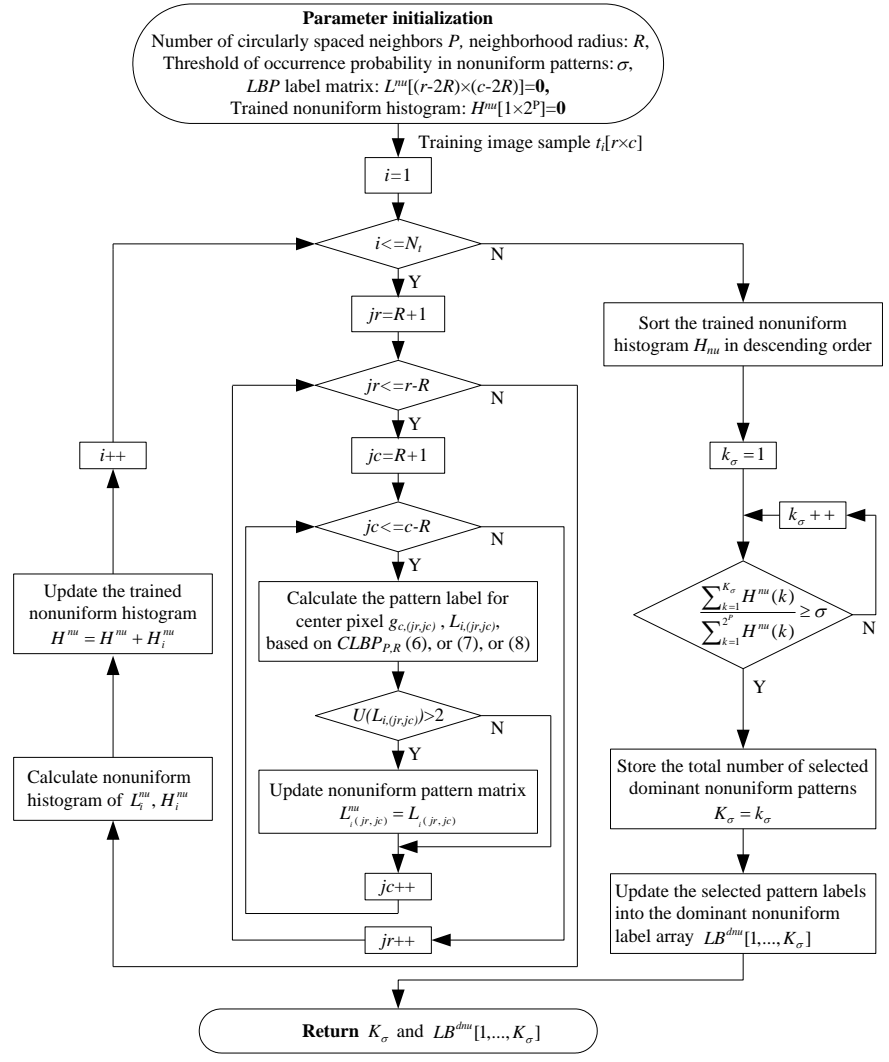

Fig. 4. Training procedure of the dominant nonuniform patterns for GCLBP.

\section{B. Hybrid Pattern Encoding}

Uniform $C L B P_{P, R}^{u 2}$, rotation invariant $C L B P_{P, R}^{r i}$ and rotation invariant uniform $C L B P_{P, R}^{r \text { rit2 }}$ are built for code mapping for the classical CLBP. Here, a novel mapping scheme, GCLBP $P_{P, R}^{h r i 2}$, is established for GCLBP. The superscript reflects the hybrid rotation invariant $u$ niform patterns classified by judging the uniformity criterion (2) with 2 . The expression is given as

$$
G C L B P_{P, R}^{\text {hriu } 2}= \begin{cases}\sum_{0}^{P-1} s\left(g_{p}-g_{c}\right) & \text { when } U\left(C L B P_{P, R}\right) \leq 2 \\ C L B P_{P, R}^{r i} & \text { when } U\left(C L B P_{P, R}\right)>2 \cap C L B P_{P, R} \in L B^{d n u} \\ P+1+K_{\sigma}^{r i} & \text { when } U\left(C L B P_{P, R}\right)>2 \cap C L B P_{P, R} \notin L B^{d n u}\end{cases}
$$

where the $K_{\sigma}^{i i} \leq K_{\sigma}$ is the total number of the rotation invariant pattern codes of the selected DNUPs label set, $L B^{d n u}\left[1, \ldots, K_{\sigma}\right]$. The specific coding process is introduced as follows:

1. The exactly $P+1$ rotation invariant uniform patterns defined in the $C L B P_{P, R}$ are completely kept in the $G C L B P_{P, R}^{h r i u 2}$, the code indexes are from 0 to $P$.

2. The $K_{\sigma}$ DNUPs are labeled with $K_{\sigma}^{i j}$ rotation invariant codes using (9) (or (10)), the code indexes are from $P+1$ to $P+K_{\sigma}^{r i}$.

3. The non-DNUPs are grouped with a miscellaneous code index: $P+1+K_{\sigma}^{r i}$.

In summary, the hybrid look-up table fulfills the label mapping from $2^{P}$ raw binary codes to $P+2+K_{\sigma}^{i i}$ output codes of $G C L B P_{P, R}^{h r i u 2}$. A detailed example is presented for the ease of understanding. As shown in Fig. 5, suppose $P=8, R=1$, and the returned results are: $K_{\sigma}=9, L B^{d n u}=\{5,17,20,68,92,116,187$, $197,245\}$. The output labels applying $G C L B P_{P, R}^{\text {hriu2 }}$ are then composed of uniform part $\{0,1,2,3,4,5,6,7,8\}$, dominant nonuniform part $\{9,10,11,12,13,14\}$, and the miscellaneous nonuniform part $\{15\}$, where elements $\{5,20\}$ in $L B^{d n u}$ with the rotation invariant characteristic are jointly mapped to label 9 , analogously for elements $\{17,68\}$ and $\{92,197\}$. Notably, $K_{\sigma}^{r i}$ in this simple example decreases to 6 from the former 9 .

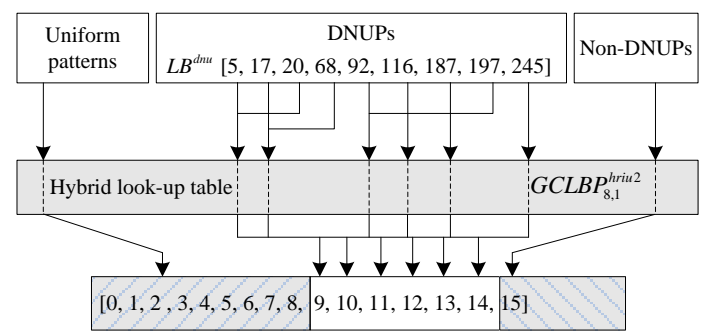

Fig. 5. Illustrative example of hybrid pattern code mapping mechanism.

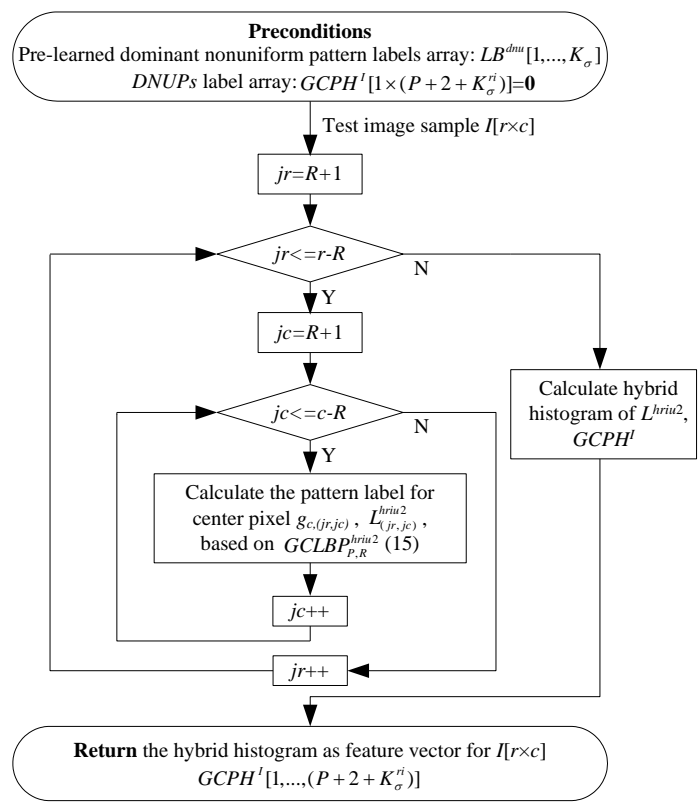

Fig. 6. Feature extraction procedure under $G C L B P_{P, R}^{\text {hriu2 }}$ framework.

\section{Feature Extraction}

Fig. 6 shows the feature extraction procedure of $G C L B P_{P, R}^{h r i u 2}$ framework in detail. First, an array buffer with a size of $1 \times\left(P+2+K_{\sigma}^{r i}\right)$ is initialized for keeping the hybrid pattern histogram. Then, for every image in the testing set, a pattern label matrix with a size of $(r-2 R) \times(c-2 R)$ is calculated for each center pixel, the hybrid histogram is calculated after all center pixels are traversed. Finally, the updated histogram $G C P H^{I}$ is returned as the GCLBP feature values of this image sample.

\section{Feature Matching}

Focusing on time efficiency, the simple nearest-neighbor classifier (NNC) is used to identify LBP-like histograms. The Chi-square distance continues to be used in this work. A test sample $\mathbf{T}=\left\{T_{i}\right\}$ to be matched will be appointed to the class model $\mathbf{M}=\left\{M_{i}\right\}$ if it occupies the minimum Chi-square distance $\chi^{2}(\mathbf{T}, \mathbf{M})=\sum_{i} \frac{\left(T_{i}-M_{i}\right)^{2}}{T_{i}+M_{i}}$

where $i=1,2, \ldots, P+2+K_{\sigma}^{r i}$, which has been defined in (15).

In addition, the multiresolution histogram matching by using multiple LBP operators with distinct $(P, R)$ is also involved in this work. 


\section{THEORY VERIFICATION}

\section{A. Characteristics of GCLBP-series Descriptors}

Two generalized properties of the GCLBP framework are outlined briefly. First, the used descriptor in Fig. 4 is not limited to original CLBP (its improved version names ICLBP). Any other CLBP-like variant such as CRLBP [36] or CNLP [25] can be strengthened to ICRLBP or ICNLP, respectively. Second, GCLBP-series descriptors completely maintain the original functions. For instance, the $I C L B P_{P, R}^{\text {hriu2 }}$ transmute themselves into $C L B P_{P, R}^{r i w 2}$ or $C L B P_{P, R}^{r i}$ when $\sigma$ is set to 0 or 1 , respectively.

Compared with DLBP, two major differences are declared here. First, GCLBP-series descriptors completely keep pattern information, i.e., only frequencies of specified patterns are extracted in ICLBP and ICNLP, while DLBP neglect pattern information. Second, GCLBP-series descriptors consider more on time-efficiency. The cyclic sorting is triggered only once under GCLBP framework but required repeatedly during the entire feature extracting process for DLBP.

\section{B. Effectiveness Verification}

Our GCLPB framework focuses on how to develop more reliable yet lightweight descriptors for feature extraction. In order to prove the effectiveness of GCLBP-series descriptors, we take a set of results (marked red in TABLE II) from the extensive tests to illustrate why the GCLBP-based descriptor, $I C L B P_{P, R}^{h r i u 2}$, can obtain outstanding performance.

In several false positives triggered by $C L B P_{P, R}^{r i u 2}$ and $C L B P_{P, R}^{r i}$, we randomly choose the classification process of Class ${ }_{16}$ for a detailed illustration. As shown in Fig. 7, $C L B P_{P, R}^{r i u 2}$ and $C L B P_{P, R}^{r i}$ always assign $\mathrm{TeS}_{1129}$ (belongs to Class ${ }_{16}$ ) to Class ${ }_{12}$ incorrectly, while our ICLBP $P_{P, R}^{\text {hriu2 }}$ rarely make this kind of mistakes. And indeed, the intra-class and inter-class challenges among Class 16 and Class 12 are quite evident.

Fig. 8 presents the histogram distributions and the specific classification results of the $\mathrm{TeS}_{1129}$ by using $C L B P_{8,3}^{r i u 2}, C L B P_{8,3}^{r i}$,
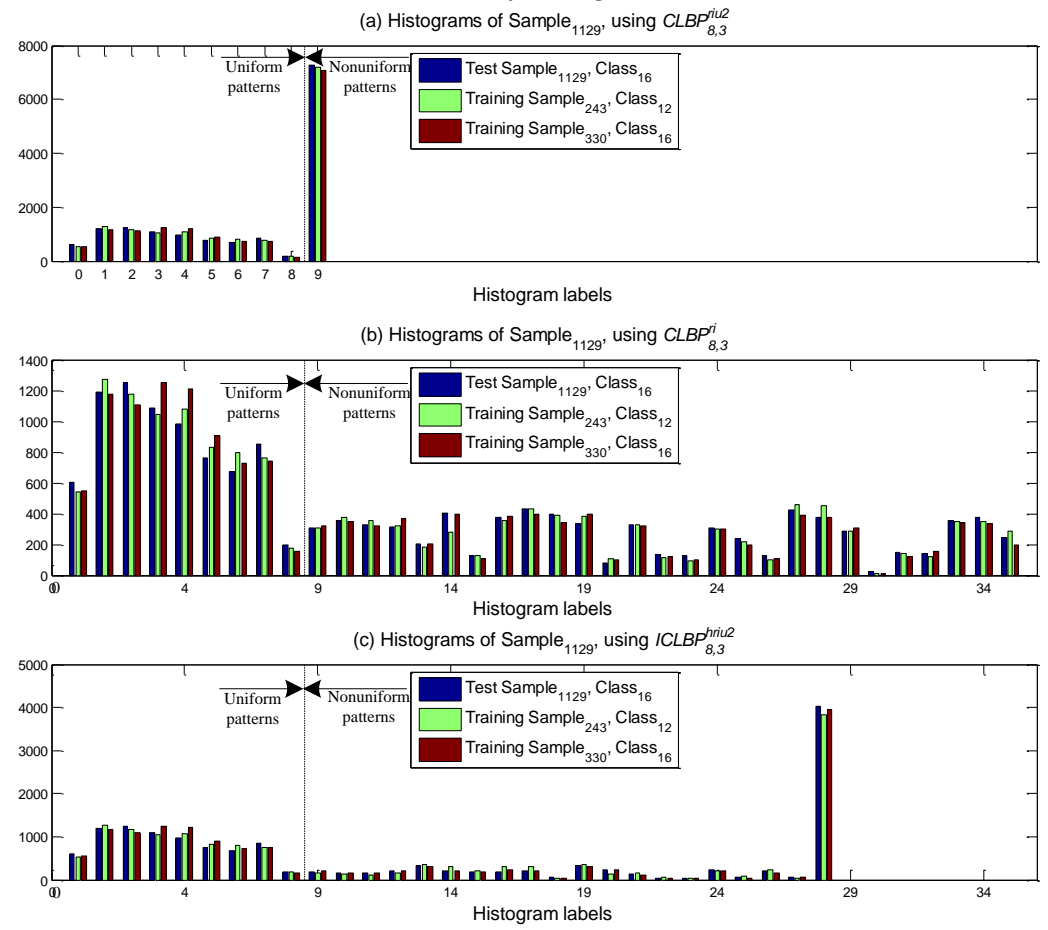

Fig. 8. Comparative histograms (a), (b), (c), and Chi-square distances (d), (e), (f) when using $C L B P_{8,3}^{r i u 2}, C L B P_{8,3}^{r i}$ and $I C L B P_{8,3}^{h r i u 2}$. and $I C L B P_{8,3}^{h r i u 2}$. For $C L B P_{8,3}^{\text {riu2 }}$, mainly based on uniform patterns (bar 0 8 in Fig. 8(a)), the minimum Chi-square distance of $\mathrm{TeS}_{1129}$ to those of 480 training samples among all 24 classes falls to the erroneous Class 12 (refer to Fig. 8(d)). The reason can be clearly observed in Fig. 8(a) that the histogram bins of $\mathrm{TeS}_{1129}$ (blue) are more similar to those of $\mathrm{TrS}_{243}$ (green) than to those of $\operatorname{TrS}_{330}$ (red). $C L B P_{8,3}^{r i}$ can suppress this drawback to some extent by adding all the rotation invariant nonuniform patterns for feature matching (refer to Fig. 8(b)), the average classification rate is then promoted from $85.21 \%$ of $C L B P_{8,3}^{\text {riu }}$ to 92.92\% (refer to TABLE II). However, as shown in Fig. 8(e), since the rotation invariance of nonuniform patterns (especially of non-DNUPs) is dramatically degenerated, $C L B P_{8,3}^{r i}$ still can inexplicitly recognize the fuzzy appearances in Fig. 7. In addition, the extra feature matching on non-DNUPs in $C L B P_{8,3}^{r i}$ are inefficient and expensive. In the training procedure in Fig. 4, the extremely noisy non-DNUPs are decisively discarded as early as they are in raw pattern codes. Thus, although the feature dimension is more lightweight than that of $C L B P_{8,3}^{r i}(29$ vs. 36), our $I C L B P_{8,3}^{\text {hriu2 }}$ can correctly identify the $\mathrm{TeS}_{1129}$ to Class $_{16}$ (refer to Fig. 8(f)). It is demonstrated clearly in Fig. 8(c) that the histogram similarity of intra-class samples have been reliably compensated by the statistically trained DNUPs, so then, the most intrinsic texture natures get fully respects.

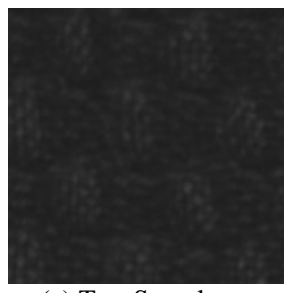

(a) Test Sample S129 $_{11}$ $\left(\mathrm{TeS}_{1129}\right)$, in Class $_{16}$



(b) Training Sample 243 $\left(\mathrm{TrS}_{243}\right)$, in Class 12

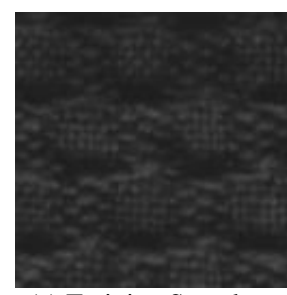

(c) Training Sample 330 $\left(\mathrm{TrS}_{330}\right)$, in Class $_{16}$
Fig. 7. Test and training image samples, (a), (b), and (c).
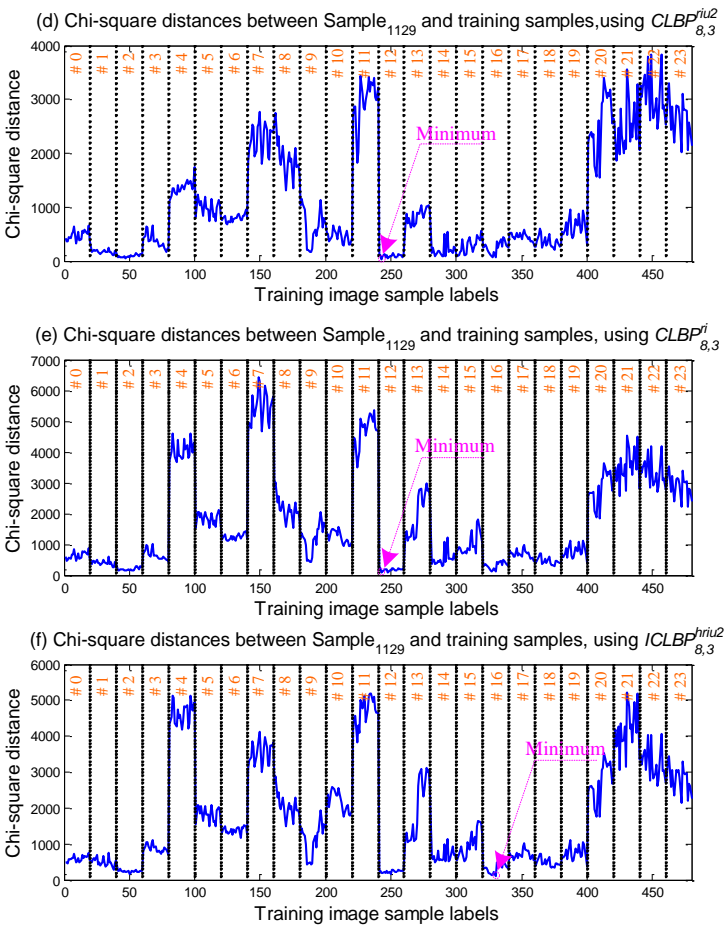


\section{EXPERIMENTS AND DISCUSSIONS}

In order to evaluate the proposed GCLBP framework, we carried out a series of experiments on two widely recognized image databases: one textile surface texture database, Outex [28], and one steel surface defect database, NEU [29].

\section{A. Results on Outex Database}

\section{1) Experimental Suites and Implementation Details}

Similar to the experiment setups in [15] [24] [26], we select two commonly used test suites of Outex_TC_00010 (TC10) and Outex_TC_00012 (TC12). They can be downloaded from the URL: http://lagis-vi.univ-lille1.fr/datasets/outex.html. As illustrated in Table I, these two test suites include the same 24 classes of textures (refer to Fig. 9), which are captured under 3 different illuminations ('Inca', 'Horizon', and 'TL84') and 9 distinct rotation angles $\left(0^{\circ}, 5^{\circ}, 10^{\circ}, 15^{\circ}, 30^{\circ}, 45^{\circ}, 60^{\circ}, 75^{\circ}, 90^{\circ}\right)$. Intuitively, TC10 and $\mathrm{TC} 12$ focus on the rotation invariance and illumination robustness, respectively. And similar to CLBP, we discuss seven ICLBP sub-schemes in the upcoming tests, which involve two fundamental ICLBP_S, ICLBP_M, three 2-D joint ICLBP_M/C, ICLBP_S/C, ICLBP_S/M, one hybrid ICLBP_S_M/C and one 3-D joint ICLBP_S/M/C, analogously for ICNLP sub-schemes.

TABLE I

TEXTURE TEST Suites Under VARIANT ILluminATION CONDITIONS

\begin{tabular}{ccc}
\hline \hline Information & TC10 & TC12 \\
\hline Illuminants & Inca & Inca, Horizon, TL84 \\
Rotations $\left(^{\circ}\right)$ & $0,5,10,15,30,45,60,75,90$ & 0 \\
Image resolution & $128 \times 128$ pixel & $128 \times 128$ pixel \\
Number of images & 4320 & 1440 \\
Number of classes & 24 & 24 \\
Number of train images & $480\left(20 \times 24,0^{\circ}\right.$, Inca $)$ & $480\left(20 \times 24,0^{\circ}\right.$, Inca $)$ \\
Number of test images & $3840(8 \times 20 \times 24)$ & $960(2 \times 20 \times 24)$ \\
\hline \hline
\end{tabular}
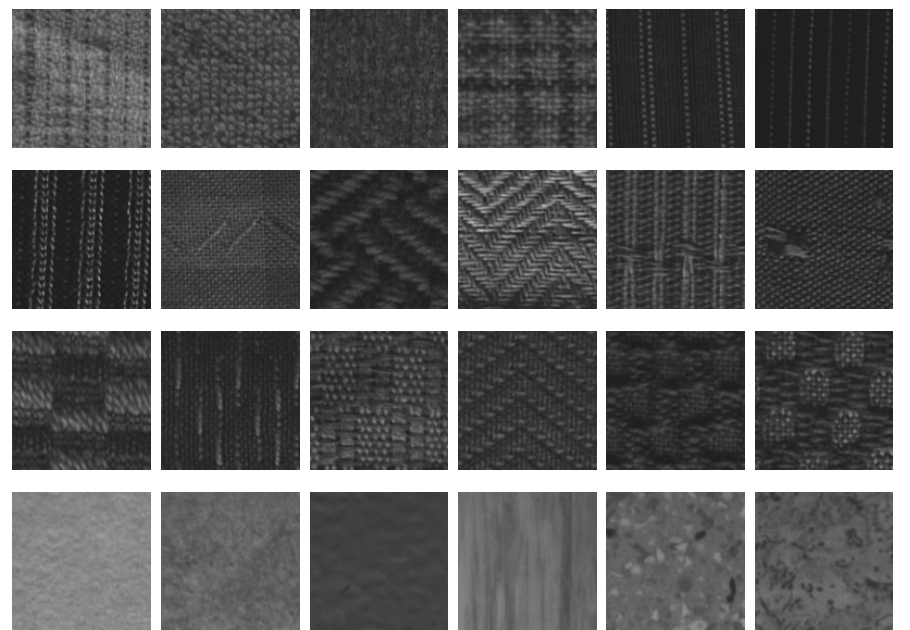

Fig. 9. The 24 classes (Class 0 - Class23) of textures on test suites TC10 and TC12.

2) Results and analysis

We discuss our experimental results in six diverse respects.

a) Ratio Threshold Learning. Considering the practical fact that surface defect inspection for hot-rolled strip steel pays more attention to illumination robustness than rotation invariance, we selected TC12 for the threshold learning tests. TABLE II lists the classification scores of the 7 ICLBP sub-schemes (column direction) under 11 evenly spaced ratio thresholds (row direction). We employed the original $C L B P_{P, R}^{r i n 2}$ and $C L B P_{P, R}^{r i}$ on both sides for baselines. From TABLE II, regardless of the value of $\sigma$, the $I C L B P_{P, R}^{\text {hriu2 }}$ variants win higher scores than both $C L B P_{P, R}^{r i t 2}$ and $C L B P_{P, R}^{r i}$. For a certain group (row) of tests, the TPR starts from the score of $C L B P_{P, R}^{r i u 2}$, then experiences stable increase to a maximum value, finally falls back to the score of $C L B P_{P, R}^{r i}$. It also proves that the $C L B P_{P, R}^{\text {riur }}$ and $C L B P_{P, R}^{r i}$ are two special cases of the proposed $I C L B P_{P, R}^{h r i u 2}$. This trend can also be observed from TABLE III (ICNLP $\left.P_{P, R}^{\text {lriph }}\right)$. Notably, before the arrival of the maximum, bigger $\sigma$ brings higher score, but triggers more DNUPs (refer to TABLE IV). While the results on the right side of table degrade gradually, which illustrates that non-DNUPs are hard to estimate reliably. Under GCLBP framework, setting $\sigma$ to 0.4-0.6 (recommend 0.5 in practice) would cover more than $90 \%$ of pattern proportion, which is also consistent with that in DLBP.

b) Classification Accuracy. For objective evaluation, we verify both the average true positive rate (TPR) and the false positive rate (FPR) for 24 classes. As illustrated in TABLE II, our ICLBP $P_{8,1}^{h r i{ }^{2} 2}$ scheme consistently exhibits higher TPRs and lower FPRs than $C L B P_{8,1}^{r i{ }^{2}}$ and $C L B P_{8,1}^{r i}$, the upward trend of TPR and the decrease trend of FPR are quite consistent. When $\sigma=0.5$, the basic $I L B P_{8,3}^{h r i u 2}$ respectively shows 12.08 and 4.37 percent better than $L B P_{8,3}^{r i u 2}$ and $L B P_{8,3}^{r i}$ on TPR, and its FPR is much lower. Further, the score of the ICLBP_S $S_{8,3}^{\text {hriu2 }} / C$ achieved $99.17 \%$, which is higher than both $93.85 \%$ of $C L B P \_S_{8,3}^{\text {ritu2 }} / C$ and 98.33\% of $C L B P \_S_{8,3}^{r i} / C$. The results of $I C N L P_{8,3}^{h r i n 2}$ in TABLE III (FPRs are omitted for space saving) are more significant than those of ICLBP $P_{8,3}^{\text {hriu }}$. These figures verify our observations claimed in Section II.D that several useful information indeed hide in the nonuniform patterns.

c) Runtime Overhead. The surface defect classification for strip steels is required to be rapid enough to satisfy the high speed hot-rolling. To simplify the table layout, we only present one representative set of tests for ICLBP $P_{8,3}^{\text {hriuz }}$ in TABLE IV, and all the results are normalized to average time per image. These measuring results are done over an Intel CPU (E3-1230-v5, 3.4 $\mathrm{GHz}$ ), with 8G RAM under Matlab R2010a platform. It is fairly clear from TABLE IV that the training time costs for DNUPs pursuing are within $45 \mathrm{~ms}$ and are needed only once, while runtime overheads for feature extraction and matching are much less. In particular, given $\sigma=0.5$, the classification time of ICLBP_ $S_{8,3}^{\text {hriu2 }} / C$ is only $2.56 \mathrm{~ms}(2.29 \mathrm{~ms}$ for feature extraction, and $0.27 \mathrm{~ms}$ for feature matching), which is better than $C L B P \_S$ ${ }_{8,3}^{r i} / C(3.56 \mathrm{~ms})$ and slightly worse than $C L B P_{-} S_{8,3}^{r i u 2} / C(1.49 \mathrm{~ms})$. However, such tiny time increase of $1.07 \mathrm{~ms}$ obtains $5.32 \%$ TPR increase over CLBP_S $S_{8,3}^{\text {riu2 }} / C$ (from $93.85 \%$ to $99.17 \%$ ). The time-saving effect will be more pronounced with bigger $P$ and $R$. Take ICLBP ${ }_{16,2}^{h r i 2}$ for an example, the total pattern dimension dramatically drops from 3166 to 212 , bringing nearly 3 times and 25 times acceleration on feature extraction and feature matching, respectively. Due to space limitation, this set of tests are omitted here. The achieved time-efficiency could be applicable for many online surface quality control applications in various manufacturing industries. 
TABLE II

Average True Positive Rate (TPR, \%) and False Positive Rate (FPR, \%) of ICLBP on TC12 When Using NNC With DifFERENT $\sigma$

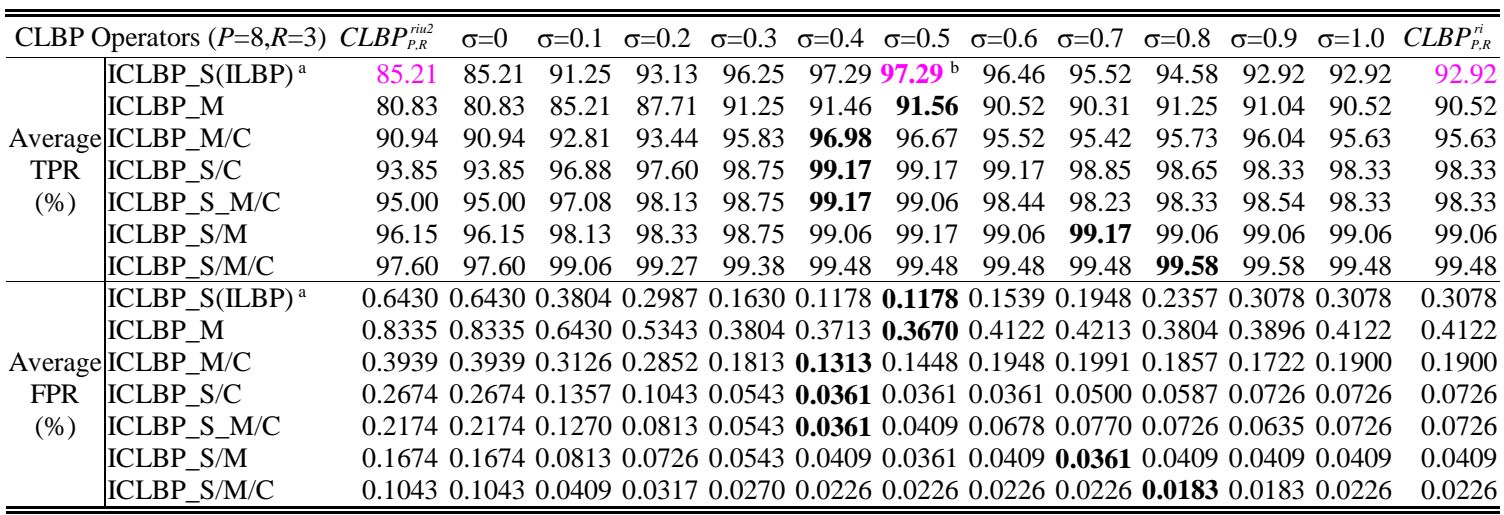

TABLE III

AVerage True Positive Rate (TPR, \%) OF ICNLP on TC12 When Using NNC WiTH DiFFeRENT $\sigma$

\begin{tabular}{|c|c|c|c|c|c|c|c|c|c|c|c|c|c|c|}
\hline \multicolumn{2}{|c|}{ CNLP Operators $(P=8, R=3$} & $C N L P_{P, R}^{r i u 2}$ & $\sigma=0$ & $\sigma=0.1$ & $\sigma=0.2$ & $\sigma=0.3$ & $\sigma=0.4$ & $\sigma=0.5$ & $\sigma=0.6$ & $\sigma=0.7$ & $\sigma=0.8$ & $\sigma=0.9$ & $\sigma=1.0$ & $C N L P_{P, R}^{r i}$ \\
\hline & ICNLP_S & 90.00 & 90.00 & 91.35 & 96.04 & 96.77 & 96.77 & 97.60 & 98.13 & 97.71 & 96.35 & 95.73 & 95.73 & 95.73 \\
\hline & ICN & 83.96 & 83.96 & 92.40 & 94.69 & 95.73 & 95.63 & 96.46 & 96.88 & 97.29 & 6.98 & 5.31 & 5.31 & 95.31 \\
\hline ver & eICNL & 5.00 & 95.00 & 96.35 & 97.50 & 98.13 & 98.54 & 98.85 & 98.75 & 98.85 & 98.54 & .23 & .33 & 8.33 \\
\hline TPR & ICNI & 83 & 95.83 & 98.65 & 99.48 & 99.48 & 58 & 58 & 99.58 & 99.69 & 69 & 9.69 & .48 & 99.48 \\
\hline$(\%)$ & ICN & 4 & 9 & 97.81 & 98.65 & 98.65 & 98.96 & 99.38 & 99.48 & 99.48 & 17 & 98.96 & 98.85 & 98.85 \\
\hline & & & 0 & 22 & 54 & 55 & 98.75 & 06 & 17 & 27 & 17 & 85 & 85 & 8.85 \\
\hline & ICNLP_S/M/C & 97.50 & 97.50 & 99.06 & 99.27 & 99.38 & 99.48 & 99.58 & 99.79 & 99.79 & 99.69 & 99.27 & 99.27 & 99.27 \\
\hline
\end{tabular}

TABLE IV

RUNTIME OVERHEADS OF ICLBP ON TC12 WHEN USING NNC WITH DIFFERENT $\sigma$

\begin{tabular}{|c|c|c|c|c|c|c|c|c|c|c|c|c|}
\hline \multicolumn{2}{|c|}{ Items $(P, R)=(8,3)$} & $\sigma=0$ & $\sigma=0$ & $\sigma=0.2$ & $\sigma=0$. & $\sigma=0$. & $\sigma=0.5$ & $\sigma=0$. & $\sigma=0$. & $\sigma=0$. & $\sigma=0$. & $\sigma=1$. \\
\hline \multicolumn{2}{|l|}{ Number of DNUPs } & 0 & 8 & 20 & 33 & 48 & 65 & 83 & 103 & 125 & 151 & 198 \\
\hline \multicolumn{2}{|c|}{ Total number of the dominant patterns } & 10 & 14 & 21 & 25 & 27 & 29 & 31 & 32 & 32 & 34 & 37 \\
\hline \multicolumn{2}{|c|}{ Average time for dominant patterns pursuing, ms } & 0.00 & 42.36 & 41.46 & 42.27 & 42.72 & 42.18 & 41.82 & 41.90 & 41.57 & 43.27 & $\overline{43.01}$ \\
\hline \multicolumn{2}{|c|}{ Average time for feature extraction, $\mathrm{ms}$} & 1.41 & 1.55 & 1.64 & 1.79 & 2.09 & 2.29 & 2.34 & 2.44 & 2.52 & 2.58 & 3.02 \\
\hline \multirow{7}{*}{$\begin{array}{l}\text { Average time for } \\
\text { feature matching, ms }\end{array}$} & ICLBP_S(ILBP) $^{a}$ & 0.07 & 0.07 & 0.08 & 0.09 & 0.14 & 0.15 & 0.15 & 0.16 & 0.16 & 0.16 & 0.19 \\
\hline & ICLBP_M & 0.06 & 0.07 & 0.07 & 0.09 & 0.13 & 0.14 & 0.14 & 0.15 & 0.15 & 0.15 & 0.19 \\
\hline & ICLBP_M/C & 0.08 & 0.13 & 0.14 & 0.16 & 0.21 & 0.33 & 0.34 & 0.32 & 0.26 & 0.34 & 0.44 \\
\hline & ICLBP_S/C & 0.08 & 0.13 & 0.14 & 0.16 & 0.21 & 0.27 & 0.26 & 0.26 & 0.26 & 0.29 & 0.54 \\
\hline & ICLBP_S_M/C & 0.14 & 0.16 & 0.17 & 0.23 & 0.28 & 0.36 & 0.33 & 0.34 & 0.34 & 0.35 & 0.75 \\
\hline & ICLBP_S/M & 0.35 & 1.22 & 1.99 & 4.10 & 7.72 & 8.85 & 9.72 & 10.32 & 10.62 & 11.75 & 15.92 \\
\hline & ICLBP_S/M/C & 1.17 & 3.68 & 5.08 & 9.81 & 17.33 & 20.91 & 23.44 & 23.60 & 25.59 & 28.17 & 35.77 \\
\hline \multicolumn{2}{|c|}{ Average classification time (ICLBP_S/C), ms } & 1.49 & 1.67 & 1.78 & 1.95 & 2.30 & 2.56 & 2.61 & 2.70 & 2.78 & 2.87 & 3.56 \\
\hline
\end{tabular}

TABLE V

AVERAge True Positive Rate (TPR, \%) ON TC12 WHEN SUfFering With VARIANT NoISE

\begin{tabular}{|c|c|c|c|c|c|c|c|}
\hline \multirow{2}{*}{$\begin{array}{c}\text { Instrument State } \\
\eta_{P S N R}\end{array}$} & \multicolumn{3}{|c|}{ Normal } & \multicolumn{2}{|c|}{ "Early Warning } & \multicolumn{2}{|c|}{ Serious Alarm } \\
\hline & 1.00 & 0.95 & 0.90 & 0.85 & 0.80 & 0.75 & $<0.75$ \\
\hline$C L B P \_S_{8,3}^{\text {ri.u2 }} / C$ & 93 & 9 & 89.69 & 76.04 & 6 & 39 & - \\
\hline$C N L P_{-}$ & 5.83 & 93 & 8 & 8 & 2 & 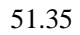 & - \\
\hline$I C L B P \_S_{8,3}^{\text {hriu2 }} / C$ & 99.17 & 97. & 96 & 90.21 & 72.15 & 54.58 & - \\
\hline$I C N L P \_S_{8,3}^{h r u 2} / C$ & 99.58 & 98.96 & 97.71 & 91.35 & 79.13 & 60.67 & - \\
\hline
\end{tabular}

${ }^{\text {a }}$ CLBP_S is essentially the original LBP operator, then ICLBP_S is the fundamental ILBP operator.

$\mathrm{b}$ The maximum classification accuracy rate of the row is emphasized with boldface.

d) Noise Robustness Performance. We continue to verify the noise robustness by adding 'Gaussian' noise artificially. A degradation metric, $\eta_{P S N R} \leq 1$, is introduced to evaluate image quality. It is the ratio of the average Peak Signal to Noise Ratio (PSNR) of degraded images to that of their standard (noise-free) training images. Fig. 10 exhibits images with different $\eta_{P S N R}$, and TABLE V lists the results of CLBP_S/C, CNLP_S/C, ICLBP_S/C and ICNLP_S/C with determined parameters of $P=8, R=3$ and $\sigma=0.5$. From TABLE V, the noise robustness of ICNLP_S/C ranks first, followed by the ICLBP_S/C. When $\eta_{P S N R}$ is no less than 0.85 , the TPRs of our sub-schemes are

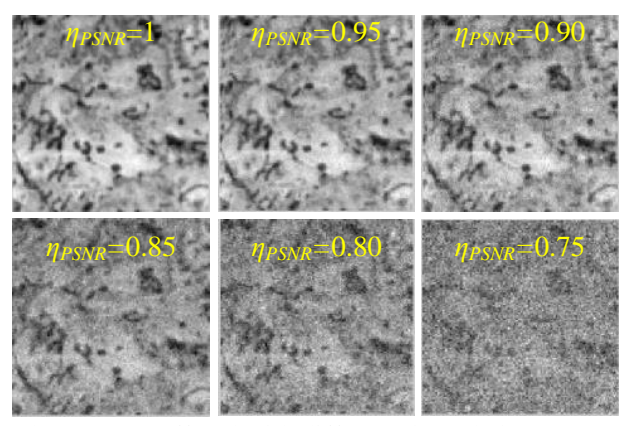

Fig. 10. The $\mathrm{TrS}_{1439}$ suffered with different degradations on PSNR. 
TABLE VI

Achieved Average True Positive Rate (TPR, \%) OF ICLBP On TC10 AND TC12 with $\sigma=0.5$



TABLE VII

COMPARATIVE Performance of ICLBP, ICNLP AND SOME STATE-OF-THE-ART Methods ON OUTEX

\begin{tabular}{|c|c|c|c|c|c|c|}
\hline \multirow[b]{2}{*}{ Method } & \multirow{2}{*}{ TC10 } & \multicolumn{2}{|c|}{ TC12 } & \multirow{2}{*}{ Mean } & \multirow{2}{*}{ Reference } & \multirow{2}{*}{$\begin{array}{c}\text { Feature } \\
\text { dimension }\end{array}$} \\
\hline & & $\mathrm{t} 184$ & horizon & & & \\
\hline$\overline{I C L B P \_S_{8,3}^{\text {hriu2 } 2}\left(I L B P_{8,3}^{\text {lriu2 } 2}\right)}$ & 86.22 & 97.66 & 96.92 & 93.60 & This paper & 29 \\
\hline ICLBP_S $S_{8,1+8,3+16,4}^{\text {hriu2 }}\left(I L B P_{8,1+8,3+16,4}^{h r i u 2}\right)$ & 95.61 & 96.68 & 95.86 & 96.05 & This paper & 351 \\
\hline$I C L B P \_S_{8,3}^{\text {hrit2 }} / C$ & 96.93 & 99.09 & 99.25 & 98.42 & This paper & 58 \\
\hline ICLBP_- $S_{8,+1,+3,3+16,4}^{\text {hriu }} / C$ & 99.59 & 99.33 & 99.21 & 99.38 & This paper & 702 \\
\hline$I C N L P \_S_{8,3}^{\text {hriu2 }} / C$ & 97.26 & 99.69 & 99.47 & 98.81 & This paper & 60 \\
\hline$I C N L P \_S_{8,1+8,3+16,4}^{\text {hrim }} / C$ & 99.51 & 99.93 & 99.87 & 99.77 & This paper & 726 \\
\hline$L_{L B P_{8,1+16,2+24,3}^{\text {riu } 2} / \text { NAR }_{8,1+16,2+24,3}[15]}$ & $97.87^{\mathrm{a}}$ & $88.42^{\mathrm{a}}$ & $86.79^{\mathrm{a}}$ & $91.02^{\mathrm{a}}$ & TPAMI 2002 & 864 \\
\hline$D L B P_{, 8,+16,2+24,3}^{r i n}[26]$ & $98.52^{\mathrm{a}}$ & $93.65^{\mathrm{a}}$ & $91.47^{\mathrm{a}}$ & $94.55^{\mathrm{a}}$ & TIP 2009 & 37 \\
\hline$C L B P \_S_{8,1+16,2+24,3}^{r \text { riu }} / M_{8,1+16,2+24,3}^{r i u 2} / C[24]$ & $99.14^{\mathrm{b}}$ & $97.60^{\mathrm{b}}$ & $98.98^{\mathrm{b}}$ & $98.57^{b}$ & TIP 2010 & 5832 \\
\hline$L T P_{8,1+16,2+24,3}^{r i u 2}[30]$ & $98.62^{\mathrm{a}}$ & $92.05^{\mathrm{a}}$ & $91.59^{\mathrm{a}}$ & $94.09^{\mathrm{a}}$ & TIP 2010 & 108 \\
\hline$C N L P \_S_{8,1+16,2+24,3}^{\text {riu } 2} M_{8,+16,2+24,3}^{\text {riu2 }} / C$ [25] & $99.12^{\mathrm{a}}$ & $98.92^{\mathrm{a}}$ & $99.08^{\text {a }}$ & $99.04 \mathrm{a}$ & MTA2016 & 5832 \\
\hline $\operatorname{dis}(S+M)_{P, R}^{\text {iniz }}[31]$ & 98.93 & 97.00 & 96.50 & 97.48 & PR 2012 & 2668 \\
\hline$N R L B P_{P, R}^{\text {ind }}[32]$ & 93.44 & 96.13 & 87.38 & 88.98 & TIP 2013 & 30 \\
\hline MSJLBP [33] & 96.67 & 95.21 & 95.74 & 95.87 & BMVC 2013 & 3540 \\
\hline PRICoLBP $P_{g}$ [34] & 94.48 & 92.57 & 92.50 & 93.18 & TPAMI 2014 & 3540 \\
\hline COV-LBPD [35] & 98.78 & 95.72 & 97.62 & 97.37 & TIP 2014 & 289 \\
\hline$M R E L B P_{P, R}^{n \text { num }}[21]$ & 99.87 & 99.49 & 99.75 & 99.70 & TIP 2016 & 800 \\
\hline
\end{tabular}

For the comparative methods, except those $\left(^{a}\right)$ which are obtained from our own implementation and those $\left({ }^{b}\right)$ which are obtained from our implementation but by using the open codes from the authors, others are directly taken from the work by L. Liu et al. [21].

stably over $90 \%$, which show significant superiorities than both CNLP_S/C and CLBP_S/C. Further, three states, normal state $\left(0.9<\eta_{P S N R} \leq 1\right)$, early-warning state $\left(0.8 \leq \eta_{P S N R} \leq 0.9\right)$, and serious alarm state $\left(\eta_{P S N R}<0.8\right)$ are recommended to be defined to enhance the reliability of steel surface inspection. Then AOI instruments will continuously send out early-warning signals in the second state, reminding operators to check and remove potential failures of related equipments (i.e., optical devices, image acquisition cards, rollers, optical-fiber cables, etc.). The last state is not allowed at any time, emergency alarm will be immediately triggered in such situation.

e) Multiresolution Configuration. As illustrated in TABLE VI, among the four single-resolution configurations, the descriptors with $(P, R)=(8,3)$ win more balanced performance, achieving competitive scores ( $98.42 \%$ for $\left.I C L B P_{-} S_{8,3}^{\text {hriu2 }} / C\right)$ and requiring nearly the smallest feature dimensions (58 for $\left.I C L B P_{-} S_{8,3}^{h r i u 2} / C\right)$. As for the multiresolution groups, although

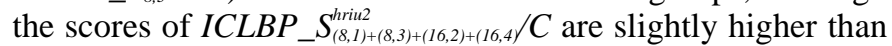
those of ICLBP_S $S_{(8,1)+(8,3)+(16,2)}^{\text {hriu2 }} / C$, the feature dimension is nearly doubled. The parameter settings of $(P, R)=(8,3)$ and $(P, R)=$ $(8,1)+(8,3)+(16,2)$ are thus recommended for single-resolution and multiresolution configuration, respectively. f) Comparative Evaluation. TABLE VII presents the comparative classification performance with those of other eleven recent state-of-the-art LBP variants on TC10 and TC12. All participant results of ICLBP are directly taken from TABLE VI (marked gray) for fair comparison. And all the subscripts and superscripts are omitted in text for simple expression. From these results, even the fundamental ILBP descriptor has effortlessly defeated the other six methods. For the rest five winners, the feature dimensions of CLBP_S/M/C, CNLP_S/M/C and $\operatorname{dis}(\mathrm{S}+\mathrm{M})$ are far larger than our ILBP. Although the recently developed COV-LBPD and MRELBP have smaller feature dimensions, our method still holds advantage in this aspect. It also can be clearly observed that $I C L B P \_S / C$ applying multiresolution scheme performs better than the first ten methods on classification scores, while the feature dimension is highly competitive to others at most of the time. Notably, our ICNLP_S/C with multiresolution scheme outperforms all other eleven LBP variants consistently, with a TPR of $99.77 \%$ and feature dimension of 726. To the best of our knowledge, the overall performance of classification accuracy, time-efficiency and application flexibility is the best report on Outex_TC10 and Outex_TC12. 


\section{B. Results on NEU Database}

\section{1) Compared Methods and Evaluation Setup}

In this section, we evaluate the classification accuracy rate and runtime overhead of two GCLBP-series descriptors on a real-world steel surface defect database, NEU. The $L B P / V A R$, $D L B P, C L B P, L T P, C N L P$, and AECLBP are selected for comparison. For fair evaluation, we adopt the identical NNC and the same encoding scheme. Also, we select the best-fit configurations suggested by their authors, i.e., $L B P_{8,1+16,2+24,3}^{\text {riu }}$ NAR $R_{8,1+16,2+24,3}, D L B P_{24,3}^{\text {riu } 2}$ with $80 \%$ dominant pattern occurrence, $C L B P \_S_{8,1+16,2+24,3}^{\text {riu2 }} / M_{8,1+16,2+24,3}^{\text {riu2 }} / C, L T P_{8,1+16,2+24,3}^{\text {riu2 }}, C N L P \_S_{8,1+16,2+24,3}^{\text {riut }} / M$ ${ }_{8,1+16,2+24,3}^{\text {riu2 }} / C, A E C L B P \_S_{8,1+16,2+24,3}^{\text {riu2 }} / M_{8,1+16,2+24,3}^{\text {riut }} / C$, and the proposed ICLBP_S $S_{8,1+8,3+16,4}^{\text {hriu2 }} / C, I C L B P \_S_{8,3}^{\text {hriu2 }} / M_{8,3}^{\text {hriu2 }} / C, I C N L P \_S_{8,1+8,3+16,4}^{\text {hriu2 }} / C$, ICNLP_S $S_{8,3}^{\text {hriu2 }} / M_{8,3}^{\text {hriu2 }} / C$ with $\sigma=0.5$. Our improved descriptors employ a smaller scope of multiresolution scheme. Even so, ours show better performance than others.

The NEU database is a recently released public database for surface defect classification of real-world hot-rolled strips. As shown in Fig. 11, the NEU database covers 6 distinct classes of typical steel surface defects, i.e., rolled-in scale (RS), patches $(\mathrm{Pa})$, crazing $(\mathrm{Cr})$, pitted surface (PS), inclusion (In), and scratches $(\mathrm{Sc})$. There are 300 non-overlapping grayscale images in each class, and the resolution of each image is $200 \times 200$ pixel. These image samples involve the first two challenges mentioned in Section I, i.e., unstable illuminations and material changes. During the evaluation, 150 samples per class are randomly selected for classifier training and the remainder ones for testing.

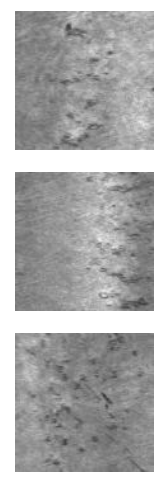

(a) RS
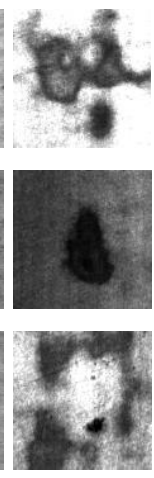

(b) $\mathrm{Pa}$
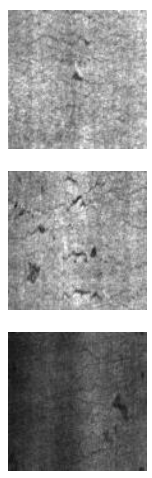

(c) $\mathrm{Cr}$
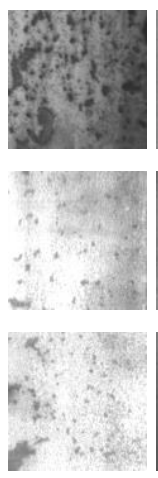

(d) PS

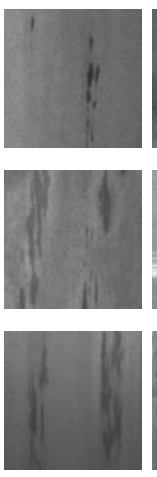

(e) In
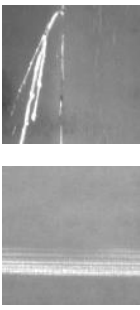

Fig. 11. The 6 classes of steel surface defects on NEU database: (a) rolled-in scale, (b) patch, (c) crazing, (d) pitted surface, (e) inclusion, and (f) scratch.

\section{2) Results and Discussion}

The average classification accuracy rates and runtime overheads carried out on NEU database are listed in TABLE VIII. Intuitively, the lightweight $D L B P$ and $L T P$ perform faster than others due to their compact feature dimensions, but exhibit lower classification scores. With a comparable time cost to $D L B P$ and $L T P$, our ICLBP_S/C and ICNLP_S/C achieved better classification scores. Benefiting from the compensation effects from trained DNUPs, their scores are then comparable with that of CLBP $(97.09 \%, 98.56 \%$ vs. $97.21 \%)$ with quite competitive runtime overheads $(76.70 \mathrm{~ms}, 90.24 \mathrm{~ms} v s .499 .25$ $\mathrm{ms})$. The noise robust $A E C L B P$ and $C N L P$ promote the classification score of $C L B P$ from $97.21 \%$ to $98.11 \%$ and $98.33 \%$, respectively. However, the time costs are slightly higher than its original $C L B P$ since they need to pay extra time on threshold re-evaluation. Further, our ICLBP $S / M / C$ and $I C N L P \_S / M / C$ with single-resolution respectively yield scores of $98.81 \%$ and $99.11 \%$, with average classification time no more than 0.3 s. Consequently, the GCLBP-series descriptors achieve balanced performance between classification accuracy and time-efficiency. In addition, multiresolution scheme involving bigger $P$ and $R$ could obtain higher classification scores, but triggers more computational expense. The relatively high time costs of $C L B P_{-} S / M / C, C N L P \_S / M / C$ and $A E C L B P \_S / M / C$ mainly result from the multiresolution scheme with a wide scale of $(8,1)+(16,2)+(24,3)$. For more insight understanding, we present the confusion matrix of the ICNLP_S $S_{8,3}^{\text {hriu2 }} / M_{8,3}^{\text {hriu2 }} / C$ in TABLE IX. It can be observed that the $\mathrm{RS}$ and $\mathrm{Cr}$ defects yields the best result with TPR of $100 \%$. Furthermore, the TPR and FPR for a certain defect type can be easily calculated from this confusion matrix.

TABLE VIII

COMPARATIVE PERFORMANCE OF ICLBP, ICNLP AND SOME STATE-OF-THE-ART METHODS ON NEU

\begin{tabular}{|c|c|c|c|c|}
\hline \multirow[b]{2}{*}{ LBP descriptors } & \multicolumn{2}{|c|}{ Accuracy } & \multicolumn{2}{|c|}{ Overheads } \\
\hline & TPR $(\%)$ & FPR $(\%)$ & $\begin{array}{c}\text { Classification } \\
\text { time }(\mathrm{ms})\end{array}$ & $\begin{array}{c}\text { Feature } \\
\text { dimension }\end{array}$ \\
\hline ICLBP_S $S_{P, R}^{\text {hriu } 2} / C$ & 97.09 & 0.58 & 76.70 & 702 \\
\hline$I C L B P \_S_{P, R}^{h r i \omega 2} / M_{P, R}^{h r i u 2} / C$ & 98.81 & 0.24 & 180.02 & 1682 \\
\hline$I C N L P \_S_{P, R}^{h r i u 2} / C$ & 98.56 & 0.29 & 90.24 & 726 \\
\hline$I C N L P \_S_{P, R}^{h r i u 2} / M_{P, R}^{h r i u 2} / C$ & 99.11 & 0.19 & 266.74 & 1800 \\
\hline$L B P_{P, R}^{r i u 2} / V A R_{P, R}[15]$ & $95.21^{\mathrm{a}}$ & $0.96^{\mathrm{a}}$ & $105.59^{a}$ & 864 \\
\hline$D L B P_{P, R}^{r i u 2}[26]$ & $94.32^{a}$ & $1.12^{\mathrm{a}}$ & $17.27^{\mathrm{a}}$ & 43 \\
\hline$C L B P \_S_{P, R}^{r i u 2} M_{P, R}^{r i u 2} / C$ [24] & $97.21^{b}$ & $0.56^{b}$ & $499.25^{b}$ & 5832 \\
\hline$L T P_{P, R}^{r i u 2}[30]$ & $95.89^{\mathrm{a}}$ & $0.82^{\text {a }}$ & $43.39^{\mathrm{a}}$ & 108 \\
\hline$C N L P \_S_{P, R}^{r i u 2} / M_{P, R}^{r i u 2} / C[25]$ & $98.33^{\mathrm{a}}$ & $0.33^{\mathrm{a}}$ & $510.54^{a}$ & 5832 \\
\hline$A E C L B P \_S_{P, R}^{r i u 2} M_{P, R}^{r i u 2} / C[8]$ & $98.11^{b}$ & $0.38^{b}$ & $524.48^{b}$ & 5832 \\
\hline
\end{tabular}

${ }^{a}$ Obtained from our own implementation.

${ }^{\mathrm{b}}$ Obtained from our implementation by using the open codes from authors.

TABLE IX

CONFUSION MATRIX OF ICNLP_ $S_{8,3}^{\text {rriu2 }} / M_{8,3}^{h \text { riu2 }} / C$ TAKEN FROM TABLE VIII

\begin{tabular}{ccccccc}
\hline \hline & RS & Pa & Cr & PS & In & Sc \\
\hline RS & 150 & 0 & 0 & 0 & 0 & 0 \\
$\mathrm{~Pa}$ & 0 & 148 & 2 & 0 & 0 & 0 \\
$\mathrm{Cr}$ & 0 & 0 & 150 & 0 & 0 & 0 \\
$\mathrm{PS}$ & 1 & 0 & 1 & 147 & 1 & 0 \\
$\mathrm{In}$ & 1 & 0 & 0 & 1 & 148 & 0 \\
$\mathrm{Sc}$ & 0 & 0 & 0 & 0 & 1 & 149 \\
\hline \hline
\end{tabular}

In summary, these preliminary results verify our initial conjecture that the descriptive information implicitly existing among the nonuniform patterns are indeed beneficial to texture classification. Our proposed GCLBP framework is precisely produced for such consideration.

\section{3) Time-efficiency Evaluation}

The GCLBP framework yields considerable classification scores. However, due to the 3-D joint scheme of histograms, the feature dimensions increase to some extent, so the runtime overheads increase accordingly. As for our AOI instrument developed in [4], the average permitted classification time per image sample $(256 \times 256$ pixel $)$ can be estimated as 
$t_{c e}=\frac{T_{\text {busy }} \times \lambda_{1}+T_{\text {idle }}}{n_{\text {dps }} \times\left(L / h_{\text {image }} \times \lambda_{2}\right)}=\frac{200 \mathrm{~s} \times 0.95+600 \mathrm{~s}}{5 \times((2000 \mathrm{~m} / 1 \mathrm{~m}) \times 0.25)}=316 \mathrm{~ms}$

where $t_{c e}$ is the ceiling of estimated time, $T_{b u s y}$ and $T_{i d l e}$ are respectively the busy and idle time slot on hot-rolling line, $\lambda_{1}$ is the acceleration factor during defect detection process, $\lambda_{2}$ is the ratio of uploaded image frames to total image frames, the size of each image frame is $1048 \times 4096$ pixel, $L$ is the average length of hot-rolled steel strip, $h_{\text {image }}$ is actual steel strip length corresponding to the height of image frame, and $n_{d p s}$ is the average estimated suspicious defect image sample among each image frame. This conservative evaluation method was formulated in long-time discussions with applied steel mills, i.e., Valin LY Steel, Baowu Steel, etc. Evidently, our developed GCLBP-based variants discussed in TABLE VIII can reliably meet this time-limited requirement. In addition, our GCLBP framework provides various options for different applications.

\section{CONCLUSION}

A novel GCLBP framework is proposed to innovatively excavate the implicit descriptive information from nonuniform patterns. Outstanding performance is achieved by the GCLBP-based descriptors on a widely used texture database. An average TPR of $99.77 \%$ is obtained by using a feature dimension of 726 . Meanwhile, an average TPR of $99.11 \%$ is obtained by the ICNLP_S/M/C on a real-world steel surface defect database within average classification time of $0.3 \mathrm{~s}$. In addition, the GCLBP framework has dual anti-noise measures: First, the noisy and phantom non-DNUPs are discarded completely. Second, GCLBP-based descriptors (such as ICNLP) perfectly inherit the noise robustness of the improved descriptors (such as CNLP). These preliminary results show that the GCLBP framework can be widely applied in the related manufacturing industries similar to hot-rolling steel productions.

However, noise suppression and algorithm acceleration are interacted and systemic issues in real-world AOI instruments. And they are also related to many other factors, for example, database integrity, hardware configurations, etc. Future work will focus on the transplantation and parallel optimization of the GCLBP framework into our previous developed AOI instrument. Besides, a test suite of steel defect database is expected to be released in the near future.

\section{ACKNOWLEDGMENT}

This work was supported by the National Natural Science Foundation of China under Grant 51704089, 51637004, the Anhui Provincial Natural Science Foundation of China under Grant 1808085QF190, the China Postdoctoral Science Foundation under Grant 2017M621996, the Fundamental Research Funds for the Central Universities of China under Grant JZ2018YYPY0296, the Ph.D Special Research Fund of HFUT under Grant JZ2016HGBZ1030, and in part by the national key research and development plan "Important Scientific Instruments and Equipment Development" under Grant 2016YFF0102200.

The authors would like to thank MVG for opening the source codes of LBP and Outex, to Z. Guo et al. for opening the source codes of CLBP, and to K. Song et al. for opening the NEU.

\section{REFERENCES}

[1] T. Sugimoto and T. Kawaguchi, "Development of a surface defect inspection system using radiant light from steel products in a hot rolling line," IEEE Trans. Instrum. Meas., vol. 47, no. 2, pp. 409-416, Apr. 1998.

[2] P. C.-Solly and J. E. Smith, "Adaptive surface inspection via interactive evolution,” Image Vis. Comput., vol. 25, no. 7, pp. 1058-1072, Jul. 2007.

[3] S. Ghorai, A. Mukherjee, M. Gangadaran and P. Dutta, "Automatic defect detection on hot-rolled flat steel products," IEEE Trans. Instrum. Meas., vol. 62, no. 3, pp. 612-621, Mar. 2013.

[4] Q. Luo and Y. He, "A cost-effective and automatic surface defect inspection system for hot-rolled flat steel," Robot. Comput.-Integr. Manuf., vol. 38, pp. 16-30, Apr. 2016.

[5] H. Kong, J. Yang and Z. Chen, "Accurate and Efficient Inspection of Speckle and Scratch Defects on Surfaces of Planar Products," IEEE Trans Ind. Informat., vol. 13, no.4, pp 1855-1865, Aug. 2017.

[6] S. Ghorai, A. Mukherjee and P. Dutta, "Discriminant analysis for fast multiclass data classification through regularized kernel function approximation," IEEE Trans. Neural Netw., vol. 21, no. 6, pp. 1020-1029, Jun. 2010.

[7] K. Liu, H. Wang, H. Chen, et al. "Steel surface defect detection using a new Haar-Weibull-Variance model in unsupervised manner. IEEE Trans. Instrum. Meas., vol. 66, no. 10, pp. 2585-2596, Oct. 2017.

[8] K. Song and Y. Yan, "A noise robust method based on completed local binary patterns for hot-rolled steel strip surface defects," Appl. Surf. Sci., vol. 285, no.21, pp. 858-864, Nov. 2013.

[9] R. Usamentiaga, D. F. Garcia, J. Molleda and F. G. Bulnes, "Vibrations in steel strips: effects on flatness measurement and filtering," IEEE Trans. Ind. Appl., vol. 50, no. 5, pp. 3103-3112, Sep. 2013.

[10] X. Gao, W. Lu, D. Tao, and X. Li, "Image quality assessment based on multiscale geometric analysis," IEEE Trans. Image Process., vol. 18, no. 7, pp. 1409-1423, Jul. 2009.

[11] F. Pernkopf, "Detection of surface defects on raw steel blocks using Bayesian network classifiers," Pattern Anal. Appl., vol. 7, no. 3, pp. 333-342, Sep. 2004.

[12] H. Hu, Y. Li, M. Liu, and W. Liang, "Classification of defects in steel strip surface based on multiclass support vector machine," Multimed. Tools Appl., vol. 69, no.1, pp. 199-216, Mar. 2014.

[13] L. Yi, G. Li and M. Jiang, "An end-to-end steel strip surface defects recognition system based on convolutional neural networks," Steel Res. Int., vol. 88, no. 2, pp.1600068: 1-12 Apr. 2016.

[14] T. Ojala, M. Pietikäinen and D. Harwood, "A comparative study of texture measures with classification based on feature distributions," Pattern Recogn., vol. 29, no. 1, pp. 51-59, Jan. 1996.

[15] T. Ojala, M. Pietikäinen and T. Mäenpää, "Multiresolution gray-scale and rotation invariant texture classification with local binary patterns," IEEE Trans. Pattern Anal. Mach. Intell., vol. 24, no. 7, pp. 971-987, Jul. 2002.

[16] G. Kylberg and I. M. Sintorn, "Evaluation of noise robustness for local binary pattern descriptors in texture classification," Eurasip J. Image Vide. Process., vol. 2013, no. 17, pp. 1-20, Dec. 2013.

[17] H. Tang, B. Yin, Y. Sun and Y. Hu, "3D face recognition using local binary patterns,' Signal Process., vol. 93, no. 8, pp. 2190-2198, Aug. 2013.

[18] T. Ahonen, A. Hadid and M. Pietikainen, "Face description with local binary patterns: application to face recognition," IEEE Trans. Pattern Anal. Mach. Intell., vol. 28, no. 12, pp. 2037-2041, Dec. 2006.

[19] G. Zhao and M. Pietikainen Dynamic, "Texture recognition using local binary patterns with an application to facial expressions," IEEE Trans. Pattern Anal. Mach. Intell., vol. 29, no. 6, pp. 915-928, Dec. 2007.

[20] R. Mehta and K. Egiazarian, "Dominant rotated local binary patterns (DRLBP) for texture classification," Pattern Recogn. Lett., vol. 71, no. 99, pp. 16-22, Feb. 2016.

[21] L. Liu, S. Lao, P. Fieguth, M. Pietikäinen, et al. "Median robust extended local binary pattern for texture classification," IEEE Trans. Image Process., vol. 25, no. 3, pp. 1368-1381, Mar. 2016.

[22] Y. Yin, X. Wang, D. Xu, F. Liu, Y. Wang and W. Wu, "Robust visual detection-learning-tracking framework for autonomous aerial refueling of UAVs," IEEE Trans. Instrum. Meas., vol. 65, no. 3, pp. 510-521, Mar. 2016.

[23] S. R. Aghdam, E. Amid and M. F. Imani, "A fast method of steel surface defect detection using decision trees applied to LBP based features," in IEEE Ind. Electron. Appl. (ICIEA), pp. 1447-1452, Jul. 2012.

[24] Z. Guo, L. Zhang and D. Zhang, "A completed modeling of local binary patternoperator for texture classification," IEEE Trans. Image Process., vol. 19 , no. 6 , pp. 1657-1663, Jun. 2010. 
[25] N. Shrivastava and V. Tyagi. "Noise-invariant structure pattern for image texture classification and retrieval," Multimed. Tools. Appl., vol. 75, no. 18, pp. 10887-10906, Sep. 2016.

[26] S. Liao, M.W. Law and A.C. Chung, "Dominant local binary patterns for texture classification," IEEE Trans. Image Process., vol. 18, no. 6, pp. 1107-1118, May 2009.

[27] E.J. Kirkland, "Bilinear interpolation. In: Advanced computing in electron microscopy," Springer, Boston, MA, 2010, pp. 261-263.

[28] T. Ojala, T. Mäenpää, M. Pietikäinen, et al. "Outex - New framework for empirical evaluation of texture analysis algorithms," in Proc. IEEE Int. Conf. Pattern Recogn., 2002, pp. 701-706.

[29] K. Song, S. Hu and Y. Yan, "Automatic recognition of surface defects on hot-rolled steel strip using scattering convolution network," J. Comput. Inf. Syst., vol. 10, no. 7, pp. 3049-3055, Apr. 2014.

[30] X. Tan and B. Triggs, "Enhanced local texture feature sets for face recognition under difficult lighting conditions," IEEE Trans. Image Process., vol. 19, no. 6, pp. 1635-1650, Jun. 2010.

[31] Y. Guo, G. Zhao, and M. Pietikäinen, "Discriminative features for texture description," Pattern Recogn., vol. 45, no. 10, pp. 3834-3843, Oct. 2012.

[32] J. Ren, X. Jiang, and J. Yuan, "Noise-resistant local binary pattern with an embedded error-correction mechanism," IEEE Trans. Image Process., vol. 22, no. 10, pp. 4049-4060, Oct. 2013.

[33] X. Qi, Y. Qiao, C. Li, and J. J. Guo, "Multi-scale joint encoding of local binary patterns for texture and material classification," in Proc. Brit. Mach. Vis. Conf. (BMVC), pp. 1-11, Sep. 2013.

[34] X. Qi, R. Xiao, C.-G. Li, Y. Qiao, J. Guo, and X. Tang, "Pairwise rotation invariant co-occurrence local binary pattern," IEEE Trans. Pattern Anal. Mach. Intell., vol. 36, no. 11, pp. 2199-2213, Nov. 2014.

[35] X. Hong, G. Zhao, M. Pietikainen, and X. Chen, "Combining LBP difference and feature correlation for texture description," IEEE Trans. Image Process., vol. 23, no. 6, pp. 2557-2568, Jun. 2014.

[36] Y. Zhao, W. Jia, R. Hu, et al. "Completed robust local binary pattern for texture classification," Neurocomputing, vol. 106, no.6, pp. 68-76, Apr. 2013.

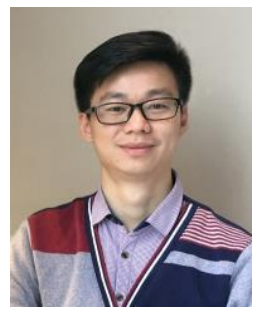

Qiwu Luo (M'17) received the B.S. degree in communication engineering from the National University of Defense Technology, Changsha, China, in 2008, the M.Sc. degree in electronic science and technology and the Ph.D. degree in electrical engineering from Hunan University, Changsha, in 2011 and 2016,

respectively.

$\mathrm{He}$ is currently a Lecturer with the School of Electrical Engineering and Automation, Hefei University of Technology, Hefei, China. He was a Senior Engineer of instrumentation with WASION Group Ltd. Company, Changsha, China, and the Deputy Technical Director with Hunan RAMON Technology Co., Ltd., Changsha, China. His research interests include the research of real-time information processing, parallel hardware architecture design and reconfigurable computing, and fault testing and diagnosis of large-scale analog circuits.

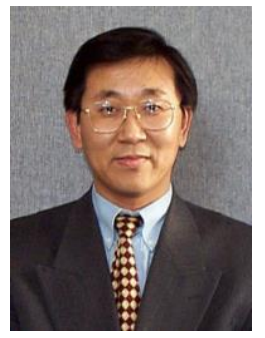

Yichuang Sun (M'90-SM'99) received the B.Sc. and M.Sc. degrees from Dalian Maritime University, Dalian, China, in 1982 and 1985, respectively, and the Ph.D. degree from the University of York, York, U.K., in 1996, all in communications and electronics engineering. He is currently a Professor and HoD with the School of Engineering and Technology of the University of Hertfordshire, UK. His research interests are mainly in the areas of wireless and mobile communications and RF and analogue circuits. He has published over 300 papers and contributed 10 chapters in edited books. He has also published 4 text and research books: Continuous-time Active Filter Design (CRC Press, USA, 1999), Design of High frequency Integrated Analogue Filters (IEE Press, UK, 2002), Wireless Communication Circuits and Systems (IET Press, 2004), and Test and Diagnosis of Analogue, Mixed-signal and RF Integrated Circuits - the Systems on Chip Approach (IET Press, 2008).

He was a Series Editor of IEE Circuits, Devices and Systems Book Series (2003-2008). He has been Associate Editor of IEEE Transactions on Circuits and Systems I: Regular Papers (2010-2011, 2016-2017, 2018-2019). He is also Editor of ETRI Journal, Journal of Semiconductors and some others. He was Guest Editor of 8 IEEE and IEE/IET journal special issues: High-frequency Integrated Analogue Filters in IEE Proc. Circuits, Devices and Systems (2000), RF Circuits and Systems for Wireless Communications in IEE Proc. Circuits, Devices and Systems (2002), Analogue and Mixed-Signal Test for Systems on Chip in IEE Proc. Circuits, Devices and Systems (2004), MIMO Wireless and Mobile Communications in IEE Proc. Communications (2006), Advanced Signal Processing for Wireless and Mobile Communications in IET Signal Processing (2009), Cooperative Wireless and Mobile Communications in IET Communications (2013), Software-Defined Radio Transceivers and Circuits for 5G Wireless Communications in IEEE Transactions on Circuits and Systems-II (2016), and IEEE International Symposium on Circuits and Systems in IEEE Transactions on Circuits and Systems-I (2016). He has also been widely involved in various IEEE technical committee and international conference activities.

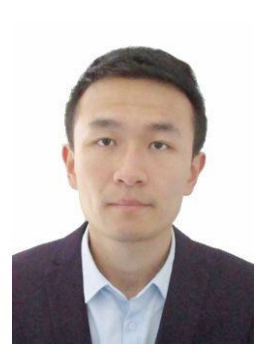

Pengcheng Li received the B.S. degree in building environment and equipment engineering from Anhui Jianzhu University, Hefei, China, in 2010, the Ph.D. degree in thermal engineering from University of Science and Technology of China, Hefei, in 2016.

$\mathrm{He}$ is currently a Lecturer with the School of Automobile and Traffic Engineering, Hefei University of Technology, Hefei, China. His field of research is solar organic Rankine cycle.

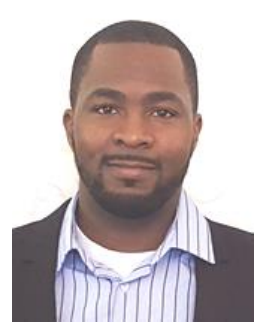

Oluyomi Simpson (M'15) received the B.Eng. (Hons) degree in electrical and electronic engineering in 2007, M.Sc. degree in radio and mobile communication systems in 2008 and Ph.D. degree in electronics engineering in 2016, all from the University of Hertfordshire, Hatfield, U.K.

$\mathrm{He}$ is currently a Lecturer in communications and electronics engineering with the School of Engineering and Technology at the University of Hertfordshire, Hatfield, U.K. His current research interests span a wide range 
of topics in radio and mobile communications, including cooperative communications, SDR, VLSI, DSP, IoT, spectrum sensing, cognitive radio, electronic testing and embedded systems.

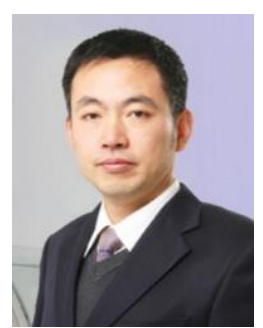

Lu Tian received the B.S. degree in physics of metals from Wuhan University, Wuhan, China, in 1983, the M.Sc. degree in physics from the Institute of Metal Research, Chinese Academy of Sciences, Liaoning, Shenyang, China, in 1988, and the $\mathrm{Ph}$. $\mathrm{D}$. degree in iron and steel metallurgy from University of Science \& Technology Beijing, Beijing, China, in 2013.

In 1993, he co-founded RAMON Science \& Technology Co. Ltd., where he is currently the President of the company. He is also a Guest Professor with the Powder Metallurgy Research Institute, Central South University, Changsha, China. His research interests include automatic steel rolling, steel surface quality inspection, and dynamic soft reduction technology. He is a Senior Member of the academic committee of continuous casting theory and new technology, Chinese Society of Metals.

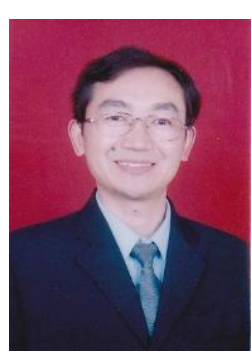

Yigang He (M'17) received the M.Sc. degree in electrical engineering from Hunan University, Changsha, China, in 1992, and the Ph.D. degree in electrical engineering from Xi'an Jiaotong University, Xi'an, China, in 1996.

In 1990, he joined the College of Electrical and Information Engineering, Hunan University, and was promoted to Associate Professor in 1996 and a Professor in 1999. From 2006 to 2011, he was the Director of the Institute of Testing Technology for Circuits and Systems, Hunan University. He was a Senior Visiting Scholar with the University of Hertfordshire, Hatfield, U.K., in 2002. From 2011 to 2017, he was the HoD with the School of Electrical Engineering and Automation, Hefei University of Technology, China. He is currently a Vice-HoD with the School of Electrical Engineering, Wuhan University, Wuhan, China. He has authored some 200 journal and conference papers in the aforementioned areas and several chapters in edited books. His current research interests include circuit theory and its applications, testing and fault diagnosis of analog and mixed-signal circuits, electrical signal detection, smart grid, radio frequency identification technology, and intelligent signal processing. 